\title{
Differential diagnosis of pancreatic cystic masses with the quantitative analysis of spectral CT imaging: Initial results
}

\author{
Y. Zhang', L. Bai ${ }^{1}$, Y. Wen' ${ }^{2}$ X. Ke ${ }^{1}$, Y. Xie ${ }^{1}$, W. Zhang1, Z. Zhang3, \\ J. Zhou' ${ }^{*}$ \\ ${ }^{1}$ Department of Radiology, Lanzhou University Second Hospital, Lanzhou 730030, PR China \\ 2Interventional Radiology Department, Lanzhou University Second Hospital, Lanzhou 730030, PR China \\ ${ }^{3}$ Department of Radiology, Northwestern University, Chicago, IL 60611, USA
}

Original article
-Corresponding authors:
Junlin Zhou, M.D.,
E-mail: LZUzjl601@163.com
Revised: February 2020
Accepted: April 2020
Int. J. Radiat. Res., January 2021;
19(1): 155-165
DOI: 10.18869/acadpub.jirr.19.1.145

\begin{abstract}
Background: To retrospectively evaluate whether quantitative information derived from spectral imaging can improve the differential diagnosis of pancreatic cystic masses including pancreatic solid pseudopapillary epithelial neoplasms (SPENs), mucin- producing cysts and pseudocysts. Materials and Methods: From June 2015 to October 2017, 56 patients (22 pseudocysts, 18 mucin-producing cysts and 16 SPENs) who underwent spectral CT imaging were included in the study. Conventional characteristics and quantitative parameters were compared among the three groups. The receiver-operating characteristic curve was used to evaluate the diagnostic performance of parameters which had statistical significance among the three groups. Two radiologists diagnosed the pancreatic cystic masses blinded in consensus, without and with the information of the statistical analysis. Results: The conventional characteristics including age, contour, nodule and septum were the independent factors correlated with category. The quantitative parameters including effective-Z, slope of energy spectral curve (slope), iodine (water) concentration and calcium (water) concentration demonstrated significantly lower values in pseudocysts group when compared with mucin-producing cysts and SPENs groups. Slope in portal venous phase, threshold of less than 0.50 , was the best discriminator between pseudocysts group and mucin-producing cysts group, with a sensitivity of $95.5 \%$, and a specificity of $88.9 \%$. The best quantitative parameter for differentiate SPENs from mucin-producing cysts was the iodine (water) concentration in portal venous phase. With the knowledge of statistical analysis, the accuracy of the two radiologists increased from $78.5 \%$ to $90.9 \%$. Conclusion: Multi-parametric analysis with the combination of quantitative parameters derived from CT spectral imaging could improve the diagnostic performance.
\end{abstract}

Keywords: Pancreatic cystic masses, Spectral curve, Effective-Z, Iodine (water) concentration, Calcium (water) concentration.

\section{INTRODUCTION}

Cystic masses of the pancreas are common in the general population, and their incidence increases with age ${ }^{(1)}$. With the widespread use of cross-sectional imaging techniques, incidental cystic masses of the pancreas are frequently detected $(2,3)$. Cystic masses of the pancreas may be congenital, non-neoplastic or neoplastic, epithelial or mesenchymal, true or degenerative. Cystic neoplasms of the exocrine pancreas include the true epithelial-lined serous 
neoplasms, mucinous cystic neoplasms (MCNs), and intraductal papillary mucinous neoplasms (IPMNs), together with solid pseudopapillary epithelial neoplasms (SPENs) in which cystic change is due to degeneration. Pancreatitis -associated pseudocysts (inflammatory pancreatic fluid collections) are not considered to be true cysts owing to the absence of an epithelial component (4).

The management of the pancreatic cystic mass primarily depends on their malignant potential. Pancreatic pseudocysts have no risk of malignant transformation. Thus, non-surgical therapy or surveillance is generally warranted $(4$, 5). IPMNs and MCNs are often grouped together as "mucin-producing cysts" because they contain a thick, viscous, mucinous-type fluid. In contrast to pancreatic pseudocysts, mucin-producing cysts have malignant potential. IPMNs are classified into main-duct (MD) and branch-duct (BD) IPMNs depending on their involvement of the main pancreatic duct. Surgery is usually recommended for MD-IPMNs and MCNs in patients who are surgical candidates, as they are considered high-risk masses, and for cysts with "worrisome" features concerning malignancy (68). BD-IPMNs have much lower malignant potential, but surveillance is suggested (8). SPEN is a low-grade malignant tumor, and surgical resection would be the preferred management $(9$, 10). Therefore, differentiation of pancreatic pseudocysts (no malignant potential), mucin-producing cysts (some malignant potential) and SPENs (low-grade malignant) has important clinical implications. This is highlighted by large surgical series, in which just over $20 \%$ of patients were found to have a benign cyst, such as an SCA or a pseudocyst, whereas almost $80 \%$ of resected BD-IPMNs have either low, or intermediate-grade dysplasia, and thus, in retrospect, did not require surgical resection $(11,12)$.

The type of pancreatic cysts may be assessed based on imaging features and cyst fluid analysis pre-operatively (13). Imaging may show specific morphological features or "worrisome" signs of malignant transformation. However, the differentiation of pseudocysts, mucin-producing cysts and SPENs of the pancreas is often not possible on conventional CT images because all three can appear as simple unilocular cystic masses with variable cystic wall thickness (14). Even the high-quality MR examinations offers relatively limited sensitivity in the differentiation. Particularly small pseudocysts are difficult to differentiate from cystic tumors of the pancreas. A report from a high-volume center showed an overall preoperative diagnostic accuracy in pancreatic cysts of only $68 \%$ (15). When imaging is not diagnostic, cyst fluid analysis may be performed, which consists of cytological and fluid marker evaluation. Cytological analysis can reveal features that are specific for a certain class of cyst, but offers limited sensitivity in detecting malignant/ premalignant cysts (16). Measurement of tumour markers, particularly carcinoembryonic antigen can also be informative, but there is a wide overlap among the various classes of cyst (17). Studies have shown that the currently available cyst fluid markers are imperfect at identifying cyst type, and cannot identify the presence of high-grade dysplasia or invasive carcinoma. Moreover, cyst fluid analysis is an invasive procedure, and the fluid sample is often inadequate (18-20).

subtypes of cystic masses of the pancreas pre -operatively. In recent years, a new spectral CT imaging mode based on the rapid switching between high- and low-energy data sets from view to view was introduced to several clinical applications (21-26), including preoperative detection of insulinoma, differentiating hypervascular hepatic masses and diagnosis of pulmonary embolism etc. One of the main values of this imaging method over conventional CT is the ability to generate additional quantitative information, which contains monochromatic CT values at different energy levels from 40 to 140 $\mathrm{keV}$, iodine and water material density measurements, and effective- $Z$ values et al. Therefore, the purpose of our study was to investigate whether quantitative information derived from spectral imaging can improve the differential diagnosis of pancreatic cystic masses including SPENs, mucin-producing cysts and pseudocysts. To our knowledge, such a study has not yet been reported.

Int. J. Radiat. Res., Vol. 19 No. 1, January 2021 


\section{MATERIALS AND METHODS}

\section{Patients}

The Ethics Committee of our institution approved this retrospective study and waived the requirement for informed consent. On review of our institution's medical records, from June 2015 to October 2017, 56 patients (18 men, 38 women; mean age $46.3 \pm 13.5$ years; $14-79$ years) underwent CT examinations and were included in the study. All the patients underwent surgery after the imaging diagnosis and the final diagnosis was made by histopathological examination.

Pancreatic cystic masses other than pseudocyst, IPMN, MCNs (mucinous cystic adenoma/ adenocarcinoma) or SPEN were not included for evaluation in our study. A total of 141 patients were excluded from the study because the cysts measured under $1 \mathrm{~cm}$, too small cystic components for measurement, unsatisfactory imaging quality resulting from body movement and artifacts, and dual-phase contrast material-enhanced CT examinations were not performed using the spectral imaging mode.

\section{CT examinations}

Non-enhanced CT and two-phase contrast material-enhanced CT examinations were performed by using the Discovery CT750 HD scanner. All patients were scanned craniocaudally while in the supine position. Non-enhanced CT images were acquired following scout scans with the conventional helical scan mode at $120 \mathrm{kVp}$ tube voltage, auto-tube current with a noise index of $10 \mathrm{HU}$ and $5 \mathrm{~mm}$ section thickness. Patients were then injected with non-ionic contrast medium (iopamidol, $300 \mathrm{mg}$ iodine $/ \mathrm{ml}$; Iopamiro 300 , Shanghai BRACCO Sine Pharmaceutical, China) via antecubital venous access at a rate of 3-4 ml/ $\mathrm{s}$ for a total of $80-100 \mathrm{ml}$ during the late arterial phase (AP) and portal venous phase (PP). The scanning delay for AP imaging was determined using semiautomatic scan-triggering software (SmartPrep; GE Healthcare, Milwaukee, WI, USA). AP scanning automatically began $8 \mathrm{~s}$ after the trigger attenuation threshold (100 HU) was reached at the level of the supraceliac abdominal aorta. For PP, the delay was $35 \mathrm{~s}$ after the end of AP.

Both the AP and PP scans were performed using the spectral imaging mode with fast tube voltage switching $(0.5 \mathrm{~ms})$ between 80 and 140 $\mathrm{kVp}$ on adjacent views during a single rotation. Other imaging parameters were as follows: $0.625 \mathrm{~mm}$ collimation thickness, $50-\mathrm{cm}$ scan field of view; 630-640 mA tube current; 0.5-0.6-s rotational speed; and a helical pitch of 1.375. The CT volume dose index (CTDIvol) for the spectral CT mode was 12.7-15.6 mGy. Images were reconstructed with projection-based material decomposition software and a standard reconstruction kernel. The adaptive statistical iterative reconstruction (ASiR) algorithm was applied to suppress image noise of the decomposition images. Three types of images were reconstructed from the single spectral CT acquisition for analysis: conventional polychromatic images obtained at $140 \mathrm{kVp}$, water-, iodine- and calcium-based material-decomposition images, and monochromatic images obtained at energies ranging from 40 to 140 kilo electron voltage (keV). All images were reconstructed with 1.25-mm section thickness.

\section{Measurement}

The spectral CT image sets were loaded on an advanced workstation (AW4.6; GE Healthcare) with the Gemstone Spectral Imaging viewer (GSI viewer, GE Healthcare). GSI viewer allows the review of monochromatic energy images at a default as well as user-selectable energy levels, and the detailed analysis using material decomposition images (in our study, iodine-, and calcium- and water-based images). A radiologist with 5 years of experience, who did not know the final diagnosis of the patients, performed the quantitative measurements on the monochromatic images using GSI Viewer. Circular or elliptical regions of interest (ROIs) were drawn to encompass as much of the cystic component of the masses as possible avoiding the septum, nodule, and wall of the masses (median area $249 \mathrm{~mm}^{2}$; range $16-3799 \mathrm{~mm} 2$ ). A ROI data file was saved for each measurement, 
which contains monochromatic CT values at different energy levels from 40-140 keV (with $10 \mathrm{keV}$ increment), iodine, calcium and water material density measurements, and effective- $Z$ values. These measurements were performed at three adjacent image levels, and the average values were calculated. For all measurements, the size, shape, and position of the ROIs were kept constant between the two phases (AP and PP) by applying the copy-and-paste function. The recently introduced parameter slope of the spectrum curve was calculated by the following formula: slope $=\mid$ CT $(90 \mathrm{keV})-$ CT $(40 \mathrm{keV})$ |/50, which was used to analyze the composition and the iodine concentration in ROIs. The radiologist also recorded the following characteristics of pancreatic cystic mass on a standardized template: symptom (negative/ positive), location (head-neck/ body-tail), size (longest axis of the tumor), CT values of the cystic component before contrast enhancement, contour (smooth/ lobular/ irregular), septum (negative/ positive), nodule (negative/ positive), and calcification (negative/ positive).

\section{Conventional diagnosis}

Two radiologists (with 3 and 15 years of experience respectively) blinded to the histopathological diagnosis for each cyst, attempted to diagnose each cyst by means of consensus agreement based on the conventional CT findings via the picture archiving and communication system (PACS). With knowledge of the statistical analysis, the radiologists diagnosed these patients again together and blinded.

\section{Statistical analysis}

Chi-square test was used to evaluate the differences in the qualitative nominal parameters, including gender, symptom, location, contour, septum, nodule, and calcification among the three groups. The differences in the quantitative variables, including age, long axis of the tumor, nonenhanced CT value, Slope, iodine-water or calcium-water concentration and effective-Z in AP and PP among the three groups were evaluated with variance analysis.
Multi-parametric analysis was performed using binary logistic regression with quantitative variables obtained by spectral CT imaging.

AUC was used to evaluate the diagnostic performance for each parameter with statistically significant difference among the three groups. Traditionally, the accuracy of a diagnostic test is classified using the following criteria: AUC=0.9-1.0 (excellent), 0.8-0.9 (good), 0.7-0.8 (fair), 0.6-0.7 (poor), 0.5-0.6 (fail).

All the statistical analyses were performed using SPSS for Windows (version 20; SPSS, Chicago, IL, USA). A $p$-value of $<0.05$ was considered to indicate a statistically significant difference.

\section{RESULTS}

The demographic data and conventional imaging characteristics of the 56 patients included in our study are summarized in table 1 . Fifty-six patients were included: the average age of discovery of mucin-producing cysts (52.3 \pm 11.2 , years) were higher than that of pseudocysts $(45.2 \pm 12.5$, years), and higher than that of SPENs (36.6 \pm 17.1 , years), the difference was statistically significant $(p=0.006)$. The AUC of the age for differential diagnosis of the three groups was 0.449 , the corresponding sensitivity and specificity were 1.000 and $0.147 .9 .1 \%$ of pseudocysts, $33.3 \%$ of mucin-producing cysts, and $75.0 \%$ of SPENs had nodules. $18.2 \%$ of pseudocysts, $66.7 \%$ of mucin-producing cysts, and $37.5 \%$ of SPENs had septa. Regarding the contour of pancreatic cysts, half of pseudocysts $(11 / 22)$ were irregular, majority of mucin-producing cysts $(8 / 18)$ were lobulate and most of SPENs (9/16) were smooth. The AUC of the contour, septum, and nodule for differential diagnosis of the three groups were $0.774,0.674$, and 0.719 , respectively. With the sensitivity and specificity were 0.955 and $0.412,0.818$ and $0.529,0.909$ and 0.529 . There were no differences regarding gender, symptom, location, calcification, long axis, and non-enhanced CT value among the three groups (all $p$-values > 0.05) (table 1).

Regarding the quantitative variables of the

Int. J. Radiat. Res., Vol. 19 No. 1, January 2021 
cystic component of the mass derived from CT spectral imaging, the slope, iodine (water) concentration, calcium (water) concentration and effective- $Z$ in AP and PP were all higher in SPENs than those in mucin-producing cysts, and then those in pseudocysts (figure 1). The monochromatic CT values at 40 to $140 \mathrm{keV}$ in $\mathrm{AP}$ are higher in SPENs than that in mucinproducing cysts, and then that in pseudocysts, furthermore the differences were more prominent at low keV levels (figure 2).

The optimal thresholds of quantitative parameters in AP and PP for differentiating pseudocysts, mucin-producing cysts and SPENs are listed in table 2 and 3. The AUCs of slope, iodine (water) concentration, calcium (water) concentration and effective- $Z$ for differentiating mucin-producing cysts from pseudocysts were in AP: $0.885,0.952,0.741,0.962$ and in PP: $0.975,0.955,0.712,0.896$, respectively. With the threshold of slope in PP set to $\geq 0.5$ for diagnosing mucin-producing cysts, the sensitivity and specificity were $95.5 \%$ and $88.9 \%$ (table 2). The AUCs of slope, iodine (water) concentration, calcium (water) concentration and effective- $Z$ for differentiating SPENs from mucin-producing cysts were in AP: 0.917, 0.969, 0.549, 0.878 and in PP: 0.943,
$0.976,0.569,0.931$, respectively. With the threshold of iodine (water) concentration in PP set to $\geq 5.03100 \mathrm{ug} / \mathrm{cm} 3$ for diagnosing SPENs, the sensitivity and specificity were $100 \%$ and 93.7\% (table 3).

Multi-parametric analysis with logistic regression was performed by combining the above statistically significant parameters. The results showed that the canonical discriminant functions coefficients of slope, iodine (water) concentration, calcium (water) concentration and effective- $Z$ were in AP: 868.13, 2742.64, 162.67 and 66.499; in PP: $-190.99,206.55,63.20$ and -34.09; the constant item is -11133.07 . The joint distribution map (figure 3) showed that SPENs and pseudocysts could be significantly distinguished from each other. However, there was overlap between mucin-producing cysts and pseudocysts, as there was between SPENs and mucin-producing cysts. The discriminant function could predict the type of pancreatic cystic masses to some extent.

With the knowledge of statistical analysis, the accuracy of the two radiologists increased from $78.5 \%$ to $90.9 \%$. There was statistically significant difference regarding accuracy between the first and second diagnosis $(p<0.001)$.

Table 1. Demographic data and conventional imaging features of 56 patients with pseudocysts, mucin-producing cysts and SPENs.

\begin{tabular}{|c|c|c|c|c|c|}
\hline Characteristics & $\begin{array}{c}\text { Pseudocyst } \\
(\mathbf{n}=\mathbf{2 2})\end{array}$ & $\begin{array}{c}\text { Mucin-producing } \\
\text { cysts (n=18) }\end{array}$ & SPEN(n=16) & $p$-Value & AUC \\
\hline Gender & $\mathrm{F} / \mathrm{M}: 13 / 9$ & $\mathrm{~F} / \mathrm{M}: 12 / 6$ & $\mathrm{~F} / \mathrm{M}: 13 / 3$ & $0.349(\mathrm{X} 2=2.103)$ & - \\
\hline Age (mean $\mathbf{n}$ standard deviation), years & $45.2 \pm 12.5$ & $52.3 \pm 11.2$ & $36.6 \pm 17.1$ & $0.006(\mathrm{~F}=5.689)$ & 0.499 \\
\hline Symptom (positive) & $20 / 2(290.9 \%)$ & $14 / 18(77.8 \%)$ & $10 / 16(62.5 \%)$ & $0.108(\chi 2=4.450)$ & - \\
\hline Location (head-neck/body-tail) & $6 / 16$ & $9 / 9$ & $10 / 6$ & $0.094(\chi 2=4.721)$ & - \\
\hline Contour (smooth/lobulate/irregular) & $10 / 1 / 11$ & $7 / 8 / 3$ & $9 / 6 / 1$ & $0.005(\chi 2=15.034)$ & 0.774 \\
\hline Septum (positive) & $4 / 22(18.2 \%)$ & $12 / 18(66.7 \%)$ & $6 / 16(37.5 \%)$ & $0.007(\chi 2=9.787)$ & 0.674 \\
\hline Nodule (positive) & $2 / 22(9.1 \%)$ & $6 / 18(33.3 \%)$ & $12 / 16(75.0 \%)$ & $0.000(\chi 2=17.592)$ & 0.719 \\
\hline Calcification (positive) & $2 / 22(9.1 \%)$ & $6 / 18(33.3 \%)$ & $6 / 16(37.5 \%)$ & $0.083(\chi 2=4.972)$ & - \\
\hline Long axis (cm) & $70.4 \pm 48.3$ & $55.6 \pm 28.7$ & $59.3 \pm 33.0$ & $0.456(\mathrm{~F}=0.796)$ & - \\
\hline Non-enhanced CT value, HU & $9.5 \pm 4.3$ & $10.3 \pm 7.9$ & $13.1 \pm 8.1$ & $0.175(\mathrm{~F}=1.782)$ & - \\
\hline
\end{tabular}



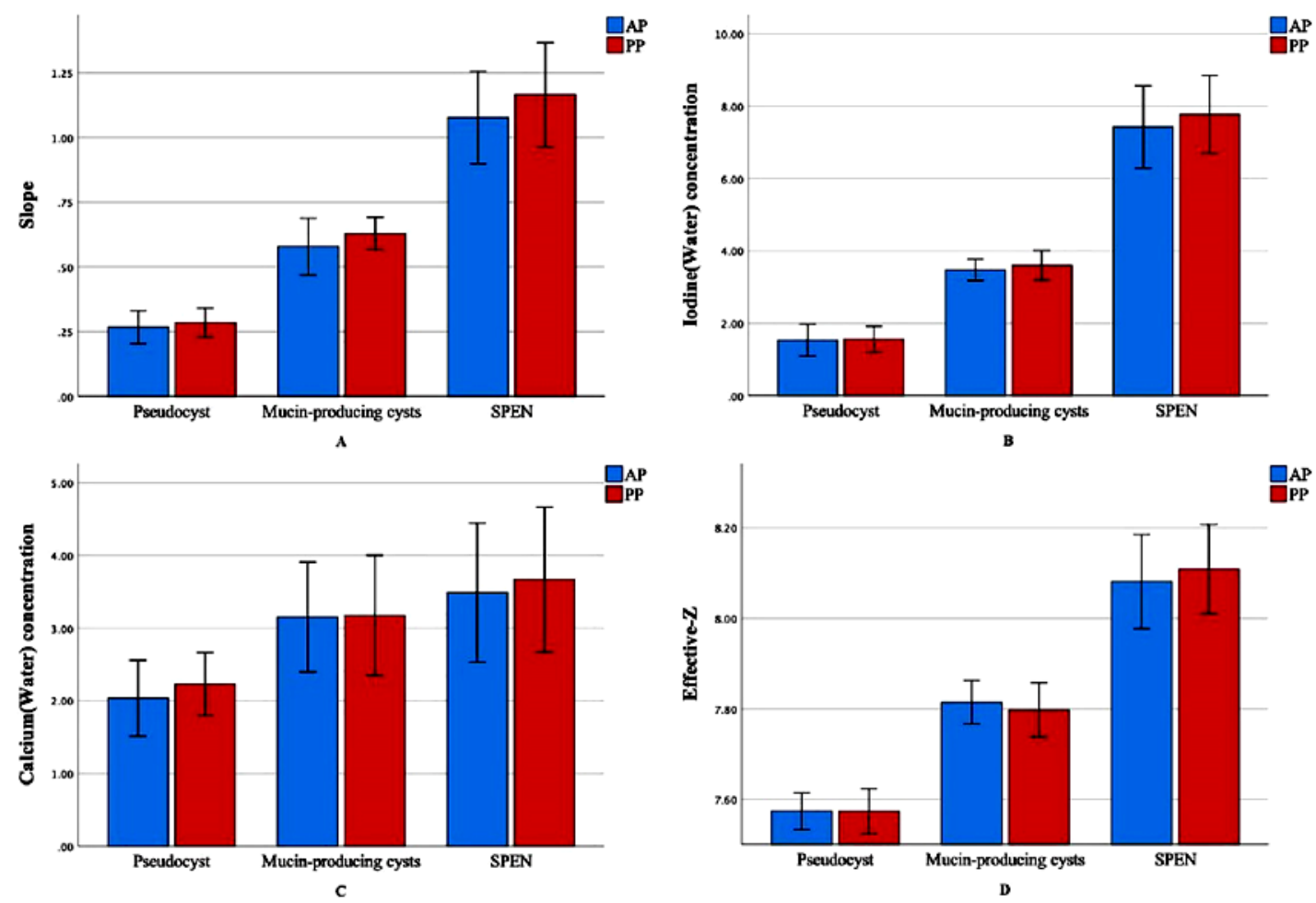

Figure 1. Bar-charts of the quantitative parameters of slope (A), iodine (water) concentration (B), calcium (water) concentration (C), and effective-Z (D), which indicate pseudocysts, mucin-producing cysts, and SPENs.

\section{Spectral HU Curves}

Figure 2. Graph depicts the average CT spectral radiodensity (in Hounsfield units) curves of pseudocysts $(n=22)$, mucin-producing cysts $(n=18)$ and SPENs $(n=16)$ of the pancreas in AP. The monochromatic CT values at 40 to 90

$\mathrm{keV}$ are significantly higher in SPENs than that in

mucin-producing cysts, and then that in pseudocysts.

Table 2. The optimal thresholds of quantitative parameters in AP and PP for differentiating mucin-producing cysts from pseudocysts.

\begin{tabular}{|c|c|c|c|c|}
\hline Group (1,2) & AUC & Threshold & $\begin{array}{c}\text { Sensitivity } \\
\text { (\%) }\end{array}$ & $\begin{array}{c}\text { Specificity } \\
\text { (\%) }\end{array}$ \\
\hline AP & & & & \\
\hline Slope & 0.885 & 0.44 & 90.9 & 88.9 \\
\hline lodine(Water) & 0.952 & 2.79 & 90.9 & 88.9 \\
\hline Calcium(Water) & 0.741 & 3.54 & 90.9 & 66.1 \\
\hline Effective-Z & 0.962 & 7.76 & 100.0 & 77.8 \\
\hline PP & & & & \\
\hline Slope & 0.975 & 0.50 & 95.5 & 88.9 \\
\hline Iodine(Water) & 0.955 & 2.56 & 90.9 & 88.9 \\
\hline Calcium(Water) & 0.712 & 2.55 & 77.3 & 72.2 \\
\hline Effective-Z & 0.896 & 7.68 & 81.8 & 88.9 \\
\hline
\end{tabular}

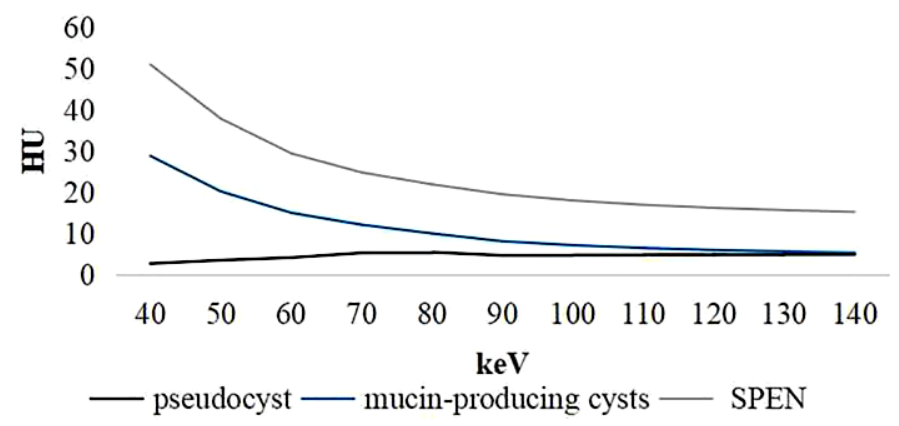

Table 3. The optimal thresholds of quantitative parameters in AP and PP for differentiating SPENs from mucin-producing cysts.

\begin{tabular}{|c|c|c|c|c|}
\hline Group (2,3) & AUC & Threshold & $\begin{array}{c}\text { Sensitivity } \\
\text { (\%) }\end{array}$ & $\begin{array}{c}\text { Specificity } \\
\text { (\%) }\end{array}$ \\
\hline AP & & & & \\
\hline Slope & 0.917 & 0.79 & 83.3 & 81.2 \\
\hline lodine (Water) & 0.969 & 4.16 & 88.9 & 93.7 \\
\hline Calcium (Water) & 0.549 & 5.07 & 100.0 & 25.0 \\
\hline Effective-Z & 0.878 & 7.96 & 94.4 & 68.7 \\
\hline PP & & & & \\
\hline Slope & 0.943 & 0.75 & 88.9 & 81.2 \\
\hline lodine (Water) & 0.976 & 5.03 & 100.0 & 93.7 \\
\hline Calcium (Water) & 0.569 & 4.78 & 88.9 & 31.2 \\
\hline Effective-Z & 0.931 & 7.92 & 83.3 & 87.5 \\
\hline
\end{tabular}

Int. J. Radiat. Res., Vol. 19 No. 1, January 2021 


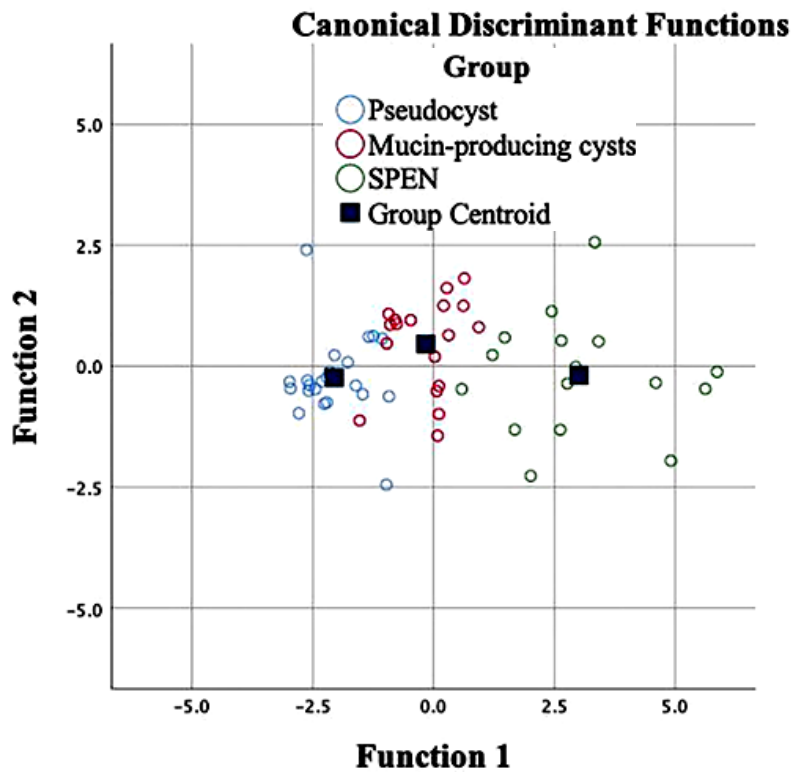

Figure 3. The joint distribution map shows that SPENs and pseudocysts could potentially be distinguished from each other. However, there are overlaps between mucin-producing cysts and pseudocysts, as there are between SPENs and mucin -producing cysts.

\section{DISCUSSION}

In our study, we focused on the use of quantitative parameters derived from CT spectral imaging to attempt to discriminate pancreatic cystic masses including SPENs, mucin-producing cysts and pseudocysts. Our results indicate that four quantitative parameters (effective-Z, slope, iodine (water) concentration and calcium (water) concentration) demonstrated statistically significantly lower values in pseudocysts group when compared with mucin-producing cysts and SPENs groups.

These results are due to different formation mechanisms of pancreatic cysts. With the cystic change of SPENs are due to degeneration or hemorrhage of neoplastic parenchyma (16), the cystic fluid of SPENs are heterogeneous and thus have higher effective- $Z$. Whereas the effective- $Z$ of pseudocysts are minimum because of they contain thin "serous"-type fluid content, including a small amount of pancreatic enzymatic secretions and inflammatory cells (6). The effective- $Z$ of mucin-producing cysts are centered because of they contain mucinous fluid with thick extracellular clean mucin(6). The energy spectral curve reflects the material CT value varying with the energy of the X-rays and the absorption characteristics to the different energy of X-rays (24). Various composition of cystic fluid exhibit changes in chemical molecular structures, and different chemical molecule structures have modified energy attenuation curves. Therefore, we can distinguish the chemical composition of cystic fluid by comparing the slope of the spectrum curve. Spectral CT imaging also generates material decomposition images so that the composition of the imaged subject can be represented as an equivalent mixture of two basis materials (for example, iodine and water) for quantitative density measurements (22). Our study demonstrated that the iodine and calcium concentration of SPENs are larger than that of mucin-producing cysts, and larger than that of pseudocysts. Yet, there were no differences regarding water concentration among the three groups in both AP and PP.

Among the quantitative parameters we compared, a slope in PP, threshold of less than 0.50 , was the best discriminator between pseudocysts group and mucin-producing cysts group, with a sensitivity of $95.5 \%$, and a specificity of $88.9 \%$. In addition, the best quantitative parameter for differentiate SPENs from mucin-producing cysts was the iodine (water) concentration in PP. The threshold of $5.03100 \mathrm{ug} / \mathrm{cm} 3$ yielded sensitivity and specificity of $100 \%$ and $93.7 \%$, respectively.

Regarding the conventional imaging features of pancreatic cystic masses, the results of our study showed that the nodule, septum and contour of pancreatic cysts were the most important characteristics in their differential diagnosis. Majority of the SPENs had nodules (figure 4), majority of the mucin-producing cysts had septa (figure 5, 6), whereas majority of the pseudocysts did not have nodules or septa (figure 7). Similar to other studies (27), our series be also showed that most of the pseudocysts were smooth or irregular, whereas most of the mucin-producing 
cysts and SPENs were smooth or lobulate. In addition, the average age of discovery of mucin-producing cysts $(52.3 \pm 11.2$, years $)$ were higher than that of pseudocysts $(45.2 \pm 12.5$, years), and higher than that of SPENs (36.6 \pm 17.1, years).
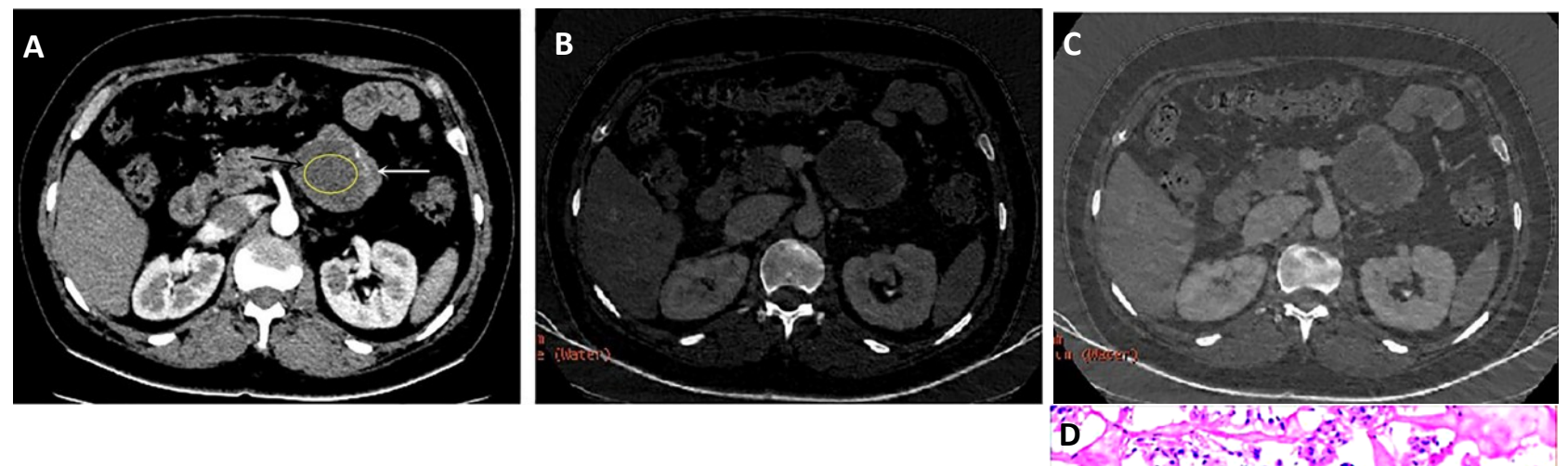

Figure 4. A 44-year-old woman with pancreatic SPEN. (A) The contrast-enhanced CT image in AP revealed a lobular cystic solid mass in the body of pancreas with the solid part mildly enhanced (white arrow). (B) lodine-based and (C) calcium-based material decomposition images generated automatically by drawing region of interest (ROI) to the cystic component of the mass (black arrow). (D) HE staining $(\times 200)$. The tumor cells were polygonal, and arranged in a pseudo-papillary shape around the axis of the fine fibrous blood vessels. Some of the nuclei were mildly heterotype.
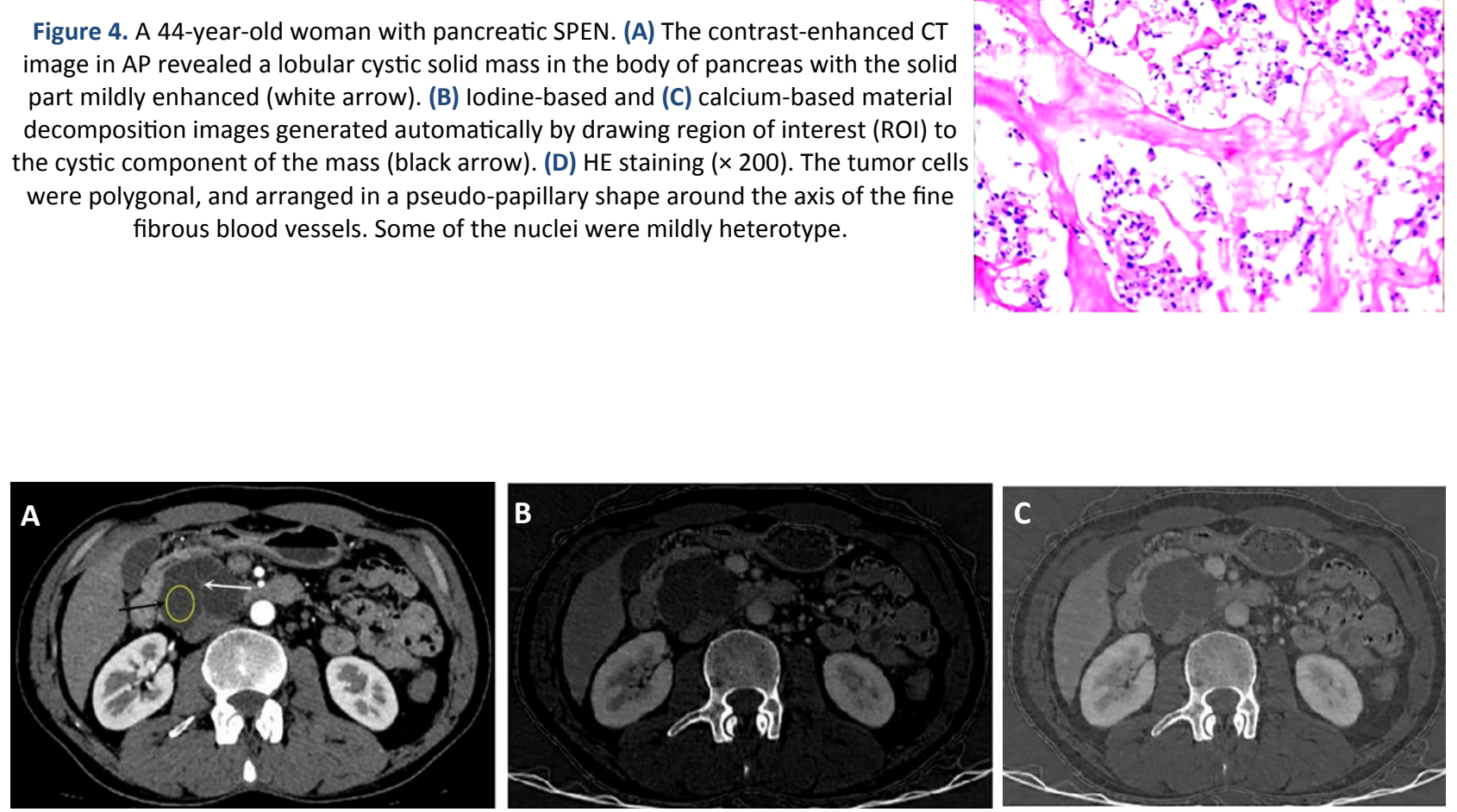

Figure 5. A 52-year-old man with pancreatic mucinous cystadenoma. (A) The contrast-enhanced CT image in AP revealed a lobular cyst in the head of pancreas with mildly enhanced septa (white arrow) in it. (B) lodine-based and (C) calcium-based material decomposition images generated automatically by drawing region of interest (ROI) to the cystic component of the mass (black arrow). (D) HE staining $(\times 200)$. The lining epithelial hyperplasia manifested as complex nipple structures, and the nuclei were multi-layered and pleomorphic.

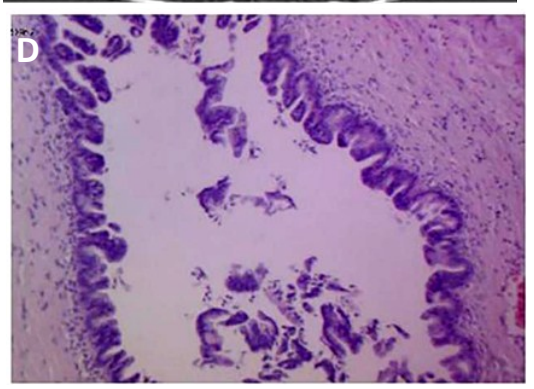



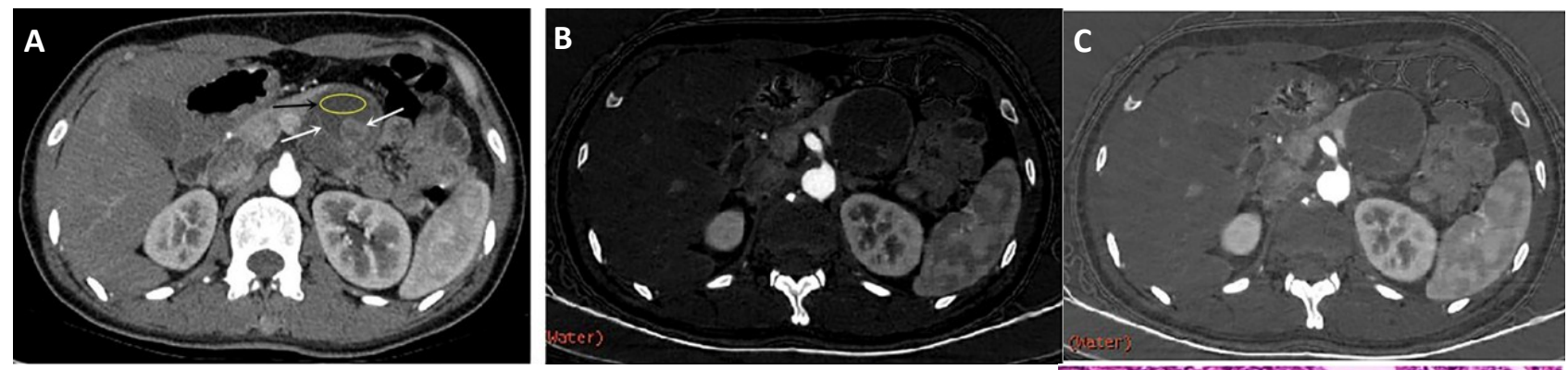

Figure 6. A 45-year-old woman with pancreatic mucinous cystadenocarcinoma. (A) The contrast-enhanced $\mathrm{CT}$ image in AP revealed a circular cyst in the body of pancreas with prominent enhanced nodule and septum (white arrow) within it. (B) lodine-based and (C) calcium-based material decomposition images generated automatically by drawing region of interest (ROI) to the cystic component of the mass (black arrow). (D) HE staining ( $\times 200)$. The tumor manifested as multiple cysts in different sizes. Some of the epithelium is papillary, local invasive growth. Nuclei enlargement, heterotype, deep staining, with mitosis.
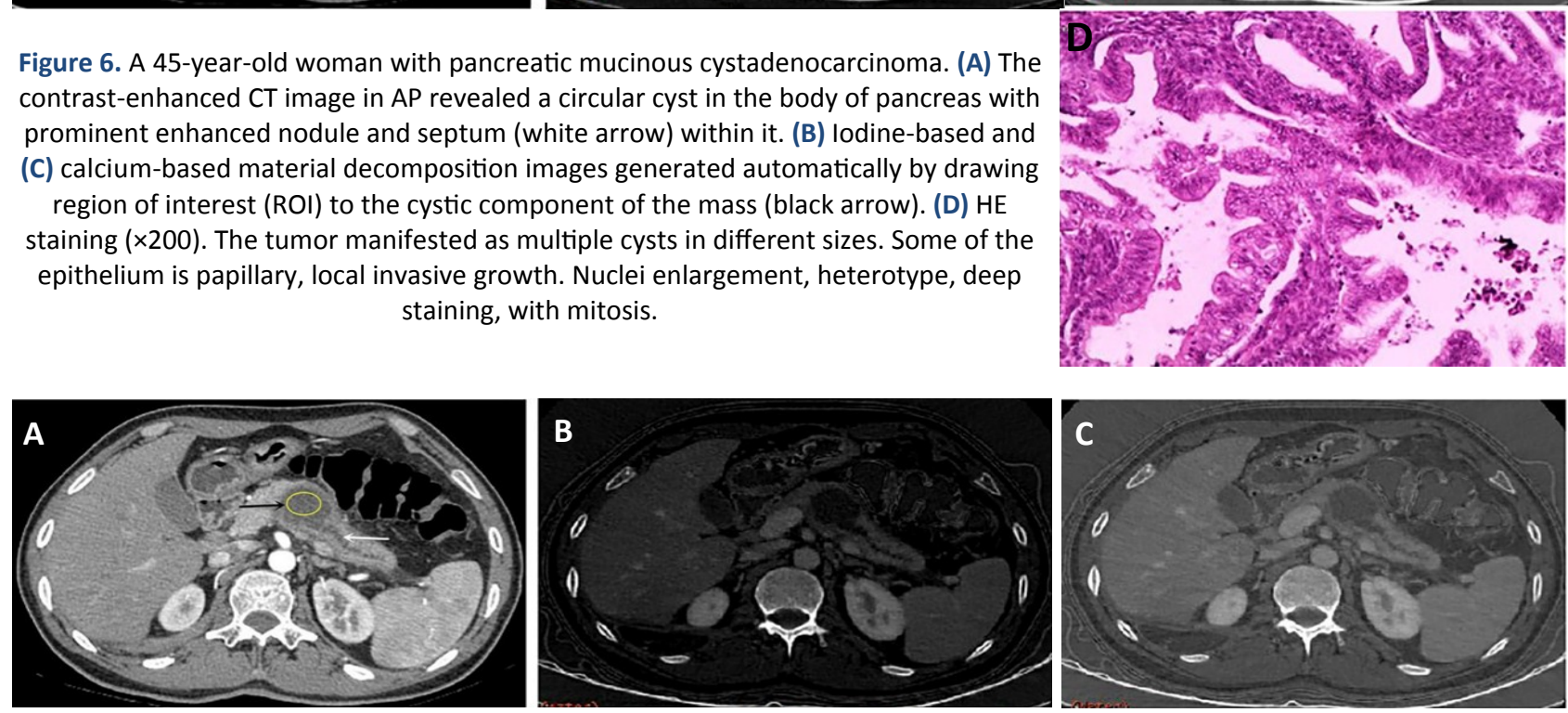

Figure 7. A 35-year-old man with pancreatic pseudocyst. (A) The contrast-enhanced CT image in AP revealed a circular cyst in the body of pancreas accompanied with dilatation of the main pancreatic duct (white arrow). (B) lodine-based and (C) calcium-based material decomposition images generated automatically by drawing region of interest (ROI) to the cyst (black arrow). (D) HE staining $(\times 100)$. There was a large amount of inflammatory cell infiltration around the remaining pancreatic tissue.

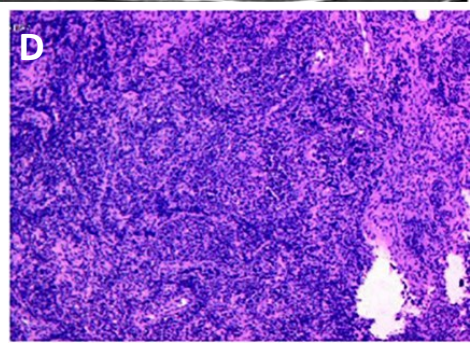

The diagnostic performance according to the general information and morphological characteristics can be variant and unstable between centers and between radiologists. At the start of our study, the diagnostic accuracy was $78.5 \%$ for the two radiologists, and twelve patients were misdiagnosed by both radiologists. With the knowledge of statistical analysis of quantitative parameters derived from CT spectral imaging, the accuracy of the two radiologists increased from $78.5 \%$ to $90.9 \%$. This result demonstrated the additional information generated from spectral CT imaging can be used to differentiate the pancreatic cystic masses.

Int. J. Radiat. Res., Vol. 19 No. 1, January 2021
Our results indicate that quantitative parameters derived from spectral imaging may have a potential role in the differentiation of (a) pseudocysts that will require non-surgical therapy from (b) SPENs or mucin-producing cysts in which surgical resection or surveillance will be suggested. Especially, our data suggest that slope and iodine (water) concentration in PP performs better in differentiating mucin-producing cysts from pseudocysts, and SPENs from mucin-producing cysts, respectively, which may reduce unnecessary surgeries. On the basis of these initial results, spectral CT imaging may be a useful noninvasive imaging tool in the differentiation of pancreatic cystic masses. In 
fact, spectral CT imaging provides all of the clinical information (eg, location, size, non-enhanced CT value, contour, septum, nodule, and calcification) of conventional CT, with the added benefit of quantitative parameters to help discriminate chemical composition of cystic fluid.

Limitations of our study included the consensus interpretation and the small number of patients evaluated. Also, there were not enough patients with MRI examination images to perform a comparison study with spectral CT imaging. The iodine (water) concentration used herein was not normalized to the iodine concentration in aorta. Hence the variation in injection rate and amount of iodinated contrast would affect the concentration of iodine on iodine (water) images. Moreover, the specimen of the cystic contents was not examined for performing an in vitro study. A larger study would be advisable to confirm these preliminary results.

In conclusion, spectral CT imaging represents a promising new imaging tool for the noninvasive differentiation of pancreatic cystic masses and can provide additional quantitative information for their diagnostic differentiation.

Ethical approval: All procedures performed in studies involving human participants were in accordance with the ethical standards of the institutional research committee and with the 1964 Helsinki declaration and its later amendments or comparable ethical standards.

\section{Conflicts of interest: Declared none.}

\section{REFERENCES}

1. GARDNER, T. B., GLASS, L. M., SMITH, K. D., RIPPLE, G. H., BARTH, R. J., KLIBANSKY, D. A., COLACCHIO, T. A., TSAPAKOS, M. J., SURIAWINATA, A. A. \& TSONGALIS, G. J. (2013) Pancreatic cyst prevalence and the risk of mucinproducing adenocarcinoma in US adults. American Journal of Gastroenterology 144, 1546-1550

2. DE JONG, K., NIO, C. Y., HERMANS, J. J., DIJKGRAAF, M. G., GOUMA, D. J., VAN EIJCK, C. H., VAN HEEL, E., KLASS, G., FOCKENS, P. \& BRUNO, M. J. (2010) High prevalence of pancreatic cysts detected by screening magnetic resonance imaging examinations. Clin Gastroenterol Hepatol 8, 806-811

3. KROMREY, M. L., BULOW, R., HUBNER, J., PAPERLEIN, C., LERCH, M. M., ITTERMANN, T., VOLZKE, H., MAYERLE, J. \& KUHN, J. P. (2018) Prospective study on the incidence, prevalence and 5-year pancreatic-related mortality of pancreatic cysts in a population-based study. Gut 67,138 145

4. FOSTER, B. R., JENSEN, K. K., BAKIS, G., SHAABAN, A. M. \& COAKLEY, F. V. (2016) Revised Atlanta Classification for Acute Pancreatitis: A Pictorial Essay. Radiographics A Review Publication of the Radiological Society of North America Inc 36, 675

5. RASCH, S., NöTZEL, B., PHILLIP, V., LAHMER, T., SCHMID, R. M. \& ALGüL, H. (2017) Management of pancreatic pseudocysts-A retrospective analysis. Plos One 12, e0184374

6. COLLINS, J. A., ALI, S. Z. \& VANDENBUSSCHE, C. J. (2016) Pancreatic Cytopathology. Surgical Pathology Clinics 9, 661

7. POZZESSERE, C., CASTAñOS GUTIéRREZ, S. L., CORONAVILLALOBOS, C. P., RIGHI, L., XU, C., LENNON, A. M., WOLFGANG, C. L., HRUBAN, R. H., GOGGINS, M. \& CANTO, M. I. (2016) Diffusion-Weighted Magnetic Resonance Imaging in Distinguishing Between Mucin-Producing and Serous Pancreatic Cysts. J Comput Assist Tomogr 40, 505-512

8. TANAKA, M., FERNáNDEZDEL, C. C., ADSAY, V., CHARI, S., FALCONI, M., JANG, J. Y., KIMURA, W., LEVY, P., PITMAN, M. B. \& SCHMIDT, C. M. (2012) International consensus guidelines 2012 for the management of IPMN and MCN of the pancreas. Pancreatology 12, 183-197

9. KEARNS, M. \& AHMAD, N. A. (2017) Diagnosis and Management of Pancreatic Cystic Neoplasms. Current Treatment Options in Gastroenterology 15, 1-16

10. 10 RADWAN, M. M., MOGHAZI, K. M., ELDEEB, N. M. F., IBRAHIM, M. E., SHARAAN, M. A. \& EMARA, D. M. (2017) $M R I$ in cystic pancreatic lesions, is it of value? Egyptian Journal of Radiology \& Nuclear Medicine 48, 771-778

11. SAHORA, K., MINOKENUDSON, M., BRUGGE, W., THAYER, S. P., FERRONE, C. R., SAHANI, D., PITMAN, M. B., WARSHAW, A. L., LILLEMOE, K. D. \& FERNANDEZDEL CASTILLO, C. F. (2013) Branch duct intraductal papillary mucinous neoplasms: does cyst size change the tip of the scale? A critical analysis of the revised international consensus guidelines in a large single-institutional series. Ann Surg 258, 466-475

12. VALSANGKAR, N. P., MORALESOYARVIDE, V., THAYER, S. P., FERRONE, C. R., WARGO, J. A., WARSHAW, A. L. \& FERNáNDEZDEL, C. C. (2012) 851 resected cystic tumors of the pancreas: a 33-year experience at the Massachusetts General Hospital. Surgery 152, S4

13. SCHEIMAN, J. M. (2017) Pancreatic Cysts - Part 1: Using the American Gastroenterological Association Guidelines for the Management of Pancreatic Cysts-A Practical Approach. Pancreas 46, 742-744

14. HENEIDY, H., YOSEF, W. \& BADR, S. (2017) Cystic pancreatic lesions; CT characterization and pathological evaluation. Egyptian Journal of Radiology \& Nuclear Medicine 48, 779-783

Int. J. Radiat. Res., Vol. 19 No. 1, January 2021 
15. CORREA-GALLEGo, C., FERRONE, C. R., THAYER, S. P., WARGO, J. A., WARSHAW, A. L. \& FERNáNDEZ-DEL, C. C. (2010) Incidental Pancreatic Cysts: Do We Really Know What We Are Watching? Pancreatology 10, 144-150

16. RIDTITID, W. \& AL-HADDAD, M. A. (2017) Endoscopic UItrasound Imaging for Diagnosing and Treating Pancreatic Cysts. Gastrointest Endosc Clin N Am 27, 615-642

17. OH, H. C., KANG, H. \& BRUGGE, W. R. (2014) Cyst fluid amylase and CEA levels in the differential diagnosis of pancreatic cysts: a single-center experience with histologically proven cysts. Dig Dis Sci 59, 3111-3116

18. AL EFISHAT, M. A., ATTIYEH, M. F., EATON, A. A., GöNEN, M., PROSSER, D., LOKSHIN, A. E., CASTILLO, C. F., LILLEMOE, K. D., FERRONE, C. R. \& PERGOLINI, I. (2018) A Multi-Institutional Validation Study of Pancreatic Cyst Fluid Protein Analysis for Prediction of High-Risk Intraductal Papillary Mucinous Neoplasms (IPMN) of the Pancreas. Annals of Surgery 268, 340-347

19. ALLEN, P. J., IACOBUZIODONAHUE, C. A. \& KLIMSTRA, D. S. (2016) Cyst Fluid Analysis in Pancreatic Intraductal Papillary Mucinous Neoplasms. Clinical Cancer Research 22, 4966

20. NGAMRUENGPHONG, S. \& LENNON, A. M. (2016) Analysis of pancreatic cyst fluid. Surg Pathol Clin 9, 677-684

21. HOU, W. S., WU, H. W., YIN, Y., CHENG, J. J., ZHANG, Q. \& $X U$, J. R. (2015) Differentiation of Lung Cancers From Inflammatory Masses with Dual-Energy Spectral CT Imaging. Acad Radiol 22, 337-344
22. LIN, X. Z., WU, Z. Y., TAO, R., GUO, Y., LI, J. Y., ZHANG, J. \& CHEN, K. M. (2012) Dual energy spectral CT imaging of insulinoma-Value in preoperative diagnosis compared with conventional multi-detector CT. European Journal of Radiology 81, 2487-2494

23. LIU, X., OUYANG, D., LI, H., ZHANG, R., LV, Y., YANG, A. \& XIE, C. (2015) Papillary thyroid cancer: dual-energy spectral CT quantitative parameters for preoperative diagnosis of metastasis to the cervical lymph nodes. Radiology 275 , 167-176

24. LV, P., LIN, X. Z., LI, J., LI, W. \& CHEN, K. (2011) Differentiation of small hepatic hemangioma from small hepatocellular carcinoma: recently introduced spectral CT method. Radiology 259, 720

25. MILETO, A., NELSON, R. C., MARIN, D., CHOUDHURY, K. R. \& HO, L. M. (2015) Dual-Energy Multidetector CT for the Characterization of Incidental Adrenal Nodules: Diagnostic Performance of Contrast-enhanced Material Density Analysis. Radiology 274, 445-454

26. SILVA, A. C., MORSE, B. G., HARA, A. K., PADEN, R. G., HONGO, N. \& PAVLICEK, W. (2011) Dual-energy (spectral) CT: applications in abdominal imaging. Radiographics A Review Publication of the Radiological Society of North America Inc 31, 1031

27. LEE, J. H., KIM, J. K., KIM, T. H., PARK, M. S., YU, J. S., CHOI, J. Y., KIM, J. H., KIM, Y. B. \& KIM, K. W. (2012) MRI features of serous oligocystic adenoma of the pancreas: differentiation from mucinous cystic neoplasm of the pancreas. $\mathrm{Br} \mathrm{J}$ Radiol 85, 571-576 
